\title{
FACE BIMAGIC LABELING ON SOME GRAPHS
}

\section{B.Roopa ${ }^{1}$, L. Shobana ${ }^{2}$}

1,2Department of Mathematics, College of Engineering and Technology, Faculty of Engineering and Technology, SRM Institute of Science and Technology, SRM Nagar, Kattankulathur-603203, Kanchipuram, Chennai, Tamil Nadu, India 1'e-mail: rb6347@ srmist.edu.in, ${ }^{2}$ e-mail: shobanal@ srmist.edu.in

Article History:Received:11 november 2020; Accepted: 27 December 2020; Published online: 05 April 2021 ABSTRACT:The existence of face bimagic labeling of types $(1,0,1),(1,1,0)$ and $(0,1,1)$ for double duplication of all vertices by edges of a ladder graph is proved. Also if $G$ is $(1,0,1)$ face bimagic, except for three sided faces then double duplication of all vertices by edges of $\mathrm{G}$ is face bimagic.

Keywords: double duplication graphs, face bimagic labeling.

\section{AMS Subject Classification: 05C78}

\section{Introduction}

In 1967 Rosa[7] introduced the concept of graph labeling. A graph labeling is assigning integers to the vertices or edges or both subject to specific conditions. Let G(V,E,F) be a graph whose vertex set, edge set and face set are $|\mathrm{V}|=\mathrm{v},|\mathrm{E}|=\mathrm{e}$ and $|\mathrm{F}|=\mathrm{f}$. A labeling of type $(\mathrm{x}, \mathrm{y}, \mathrm{z})$ of $\mathrm{G}$ assigns labels from the set $\{1,2,3, \ldots, \mathrm{xv}+\mathrm{ye}+\mathrm{zf}\}$ to vertex set, edge set and face set of $\mathrm{G}$ in such a way that each vertex will receive label $x$, each edge will receive label $y$ and each face will receive label $f$ and every label is used not more than once. The values of $\mathrm{x}, \mathrm{y}$ and $\mathrm{z}$ are restricted to $\{0,1\}$. The labelings of type $(1,0,1),(1,1,0)$ and $(0,1,1)$ represents vertex and face labelings, vertex and edge labelings and edge and face labelings respectively. The weight of a face $w_{t}(f)$ under a labeling is the sum of labels of face together with labels of vertices and edges forming that face.

Definition 1.1.[5] The double duplication of a vertex by an edge of a graph is defined as, a duplication of a vertex $\mathrm{v}_{\mathrm{k}}$ by an edge $\mathrm{e}=\left\{\mathrm{v}_{\mathrm{k}}{ }^{\prime} \mathrm{v}_{\mathrm{k}}{ }^{\prime \prime}\right\}$ in a graph $\mathrm{G}$ produces a graph $\mathrm{G}^{\prime}$ in which $\mathrm{N}\left(\mathrm{v}_{\mathrm{k}}{ }^{\prime}\right)=\left\{\mathrm{v}_{\mathrm{k}}, \mathrm{v}_{\mathrm{k}}{ }^{\prime \prime}\right\}$ and $\mathrm{N}\left(\mathrm{v}_{\mathrm{k}}{ }^{\prime \prime}\right)=\left\{\mathrm{v}_{\mathrm{k}}, \mathrm{v}_{\mathrm{k}}{ }^{\prime}\right\}$. Again duplication of vertices $\mathrm{v}_{\mathrm{k}}, \mathrm{v}_{\mathrm{k}}{ }^{\prime}$ and $\mathrm{v}_{\mathrm{k}}{ }^{\prime \prime}$ by edges $\mathrm{e}^{\prime}=\left\{\mathrm{u}_{\mathrm{k}} \mathrm{w}_{\mathrm{k}}\right\}, \mathrm{e}^{\prime \prime}=\left\{\mathrm{u}_{\mathrm{k}}{ }^{\prime} \mathrm{w}_{\mathrm{k}}{ }^{\prime}\right\}$ and $\mathrm{e}^{\prime \prime \prime}=\{$ $\left.\mathrm{u}_{\mathrm{k}}{ }^{\prime \prime} \mathrm{w}_{\mathrm{k}}{ }^{\prime \prime}\right\}$ respectively in $\mathrm{G}^{\prime}$ produces a new graph $\mathrm{G}^{\prime \prime}$ such that, $\mathrm{N}\left(\mathrm{u}_{\mathrm{k}}\right)=\left\{\mathrm{w}_{\mathrm{k}}, \mathrm{v}_{\mathrm{k}}\right\}, \mathrm{N}\left(\mathrm{w}_{\mathrm{k}}\right)=\left\{\mathrm{u}_{\mathrm{k}}, \mathrm{v}_{\mathrm{k}}\right\}$, $\mathrm{N}\left(\mathrm{u}_{\mathrm{k}}{ }^{\prime}\right)=\left\{\mathrm{w}_{\mathrm{k}}{ }^{\prime}, \mathrm{v}_{\mathrm{k}}{ }^{\prime}\right\}, \mathrm{N}\left(\mathrm{w}_{\mathrm{k}}{ }^{\prime}\right)=\left\{\mathrm{u}_{\mathrm{k}}{ }^{\prime}, \mathrm{v}_{\mathrm{k}}{ }^{\prime}\right\}, \mathrm{N}\left(\mathrm{u}_{\mathrm{k}}{ }^{\prime}\right)=\left\{\mathrm{w}_{\mathrm{k}}{ }^{\prime \prime}, \mathrm{v}_{\mathrm{k}}{ }^{\prime \prime}\right\}, \mathrm{N}\left(\mathrm{w}_{\mathrm{k}}{ }^{\prime \prime}\right)=\left\{\mathrm{u}_{\mathrm{k}}{ }^{\prime \prime}, \mathrm{v}_{\mathrm{k}}{ }^{\prime \prime}\right\}$. The double duplication of all vertices by edges of a graph $\mathrm{G}$ is denoted by $\mathrm{DD}_{\mathrm{vv}}(\mathrm{G})$.

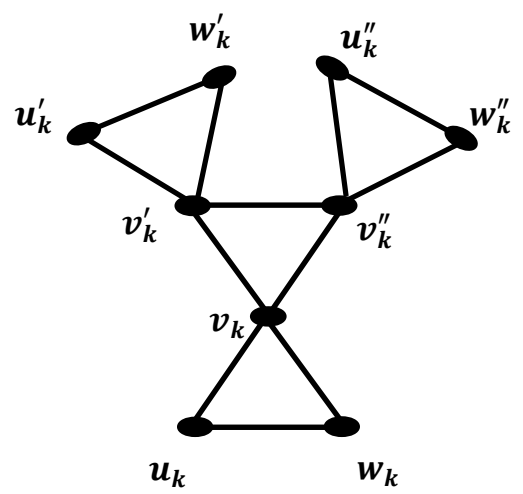

Figure 1. $\mathrm{DD}_{\mathrm{vV}}(\mathrm{G})$

Definition 1.2.[2] Let $G=(V(G), E(G), F(G))$ be a simple, finite, connected plane graph with the vertex set $\mathrm{V}(\mathrm{G})$, the edge set $\mathrm{E}(\mathrm{G})$ and the face set $\mathrm{F}(\mathrm{G})$. A bijection $\mathrm{g}$ from $\mathrm{V}(\mathrm{G}) \mathrm{UE}(\mathrm{G}) \mathrm{UF}(\mathrm{G})$ to the set $\{1,2, \ldots$, $|\mathrm{V}(\mathrm{G})|+|\mathrm{E}(\mathrm{G})|+|\mathrm{F}(\mathrm{G})|\}$ is called face bimagic if for every positive integer s the weight of every k-sided face is equal either to $\mathrm{k}_{1}$ or to $\mathrm{k}_{2}$.

${ }^{*}$ Corresponding author: B.Roopa ${ }^{1}$

Department of Mathematics, College of Engineering and Technology, Faculty of Engineering and Technology,

SRM Institute of Science and Technology, SRM Nagar,

Kattankulathur-603203, Kanchipuram, Chennai, Tamil Nadu, India

e-mail: rb6347@srmist.edu.in 


\section{Main Results}

\section{Theorem 2.1}

The graph $\mathrm{DD}_{\mathrm{VV}}\left(\mathrm{L}_{\mathrm{n}}\right), \mathrm{n} \geq 3$ of types $(1,0,1),(1,1,0)$ and $(0,1,1)$ is face bimagic.

\section{Proof:}

Let $\mathrm{G}$ be a ladder graph with vertex set $\mathrm{V}=\left\{u_{k}, u_{k}^{\prime}: 1 \leq k \leq n\right\}$, edge set $\mathrm{E}=\left\{u_{k} u_{k+1}, u_{k}^{\prime} u_{k+1}^{\prime}: 1 \leq k \leq n\right.$ $1\} \bigcup\left\{u_{k} u_{k}^{\prime}: 1 \leq k \leq n\right\}$ and face set $\mathrm{F}=\left\{l_{k}: u_{k} u_{k+1} u_{k}^{\prime} u_{k+1}^{\prime}: 1 \leq k \leq n-1\right\}$.

Let $\mathrm{G}^{\prime}$ be a graph obtained by a double duplication of a vertex by an edge in $\mathrm{G}$ with

$$
\begin{gathered}
\mathrm{V}^{\prime}=\left\{v_{k}, v_{k}^{\prime}, w_{k}, w_{k}^{\prime}, x_{k}, x_{k}^{\prime}, y_{k}, y_{k}^{\prime}, p_{k}, p_{k}^{\prime}, q_{k}, q_{k}^{\prime}, r_{k}, r_{k}^{\prime}, s_{k}, s_{k}^{\prime}: 1 \leq k \leq n\right\} \mathrm{U} \mathrm{V} \\
\mathrm{E}^{\prime}=\left\{u_{k} v_{k}, u_{k}^{\prime} v_{k}^{\prime}, u_{k} w_{k}, u_{k}^{\prime} w_{k}^{\prime}, v_{k} w_{k}, v_{k}^{\prime} w_{k}^{\prime}, u_{k} r_{k}, u_{k}^{\prime} r_{k}^{\prime}, u_{k} s_{k}, u_{k}^{\prime} s_{k}^{\prime}, r_{k} s_{k}, r_{k}^{\prime} s_{k}^{\prime},\right. \\
v_{k} x_{k}, v_{k}^{\prime} x_{k}^{\prime}, v_{k} y_{k}, v_{k}^{\prime} y_{k}^{\prime}, x_{k} y_{k}, x_{k}^{\prime} y_{k}^{\prime}, w_{k} p_{k}, w_{k}^{\prime} p_{k}^{\prime}, w_{k} q_{k}, w_{k}^{\prime} q_{k}^{\prime}, p_{k} q_{k}, p_{k}^{\prime} q_{k}^{\prime}: \\
\quad 1 \leq k \leq n\} \cup \mathrm{E} \text { and } \\
\mathrm{F}^{\prime}=\left\{f_{k}: u_{k} v_{k} w_{k}: 1 \leq k \leq n\right\} \cup\left\{g_{k}: v_{k} x_{k} y_{k}: 1 \leq k \leq n\right\} \cup\left\{h_{k}: w_{k} p_{k} q_{k}: 1 \leq k \leq n\right\} \mathrm{U} \\
\left\{z_{k}: u_{k} r_{k} s_{k}: 1 \leq k \leq n\right\} \bigcup\left\{f_{k}^{\prime}: u_{k}^{\prime} v_{k}^{\prime} w_{k}^{\prime}: 1 \leq k \leq n\right\} \bigcup\left\{g_{k}^{\prime}: v_{k}^{\prime} x_{k}^{\prime} y_{k}^{\prime}: 1 \leq k \leq n\right\} \mathrm{U} \\
\left\{h_{k}^{\prime}: w_{k}^{\prime} p_{k}^{\prime} q_{k}^{\prime}: 1 \leq k \leq n\right\} \cup\left\{z_{k}^{\prime}: u_{k}^{\prime} r_{k}^{\prime} s_{k}^{\prime}: 1 \leq k \leq n\right\} \cup \mathrm{F} .
\end{gathered}
$$

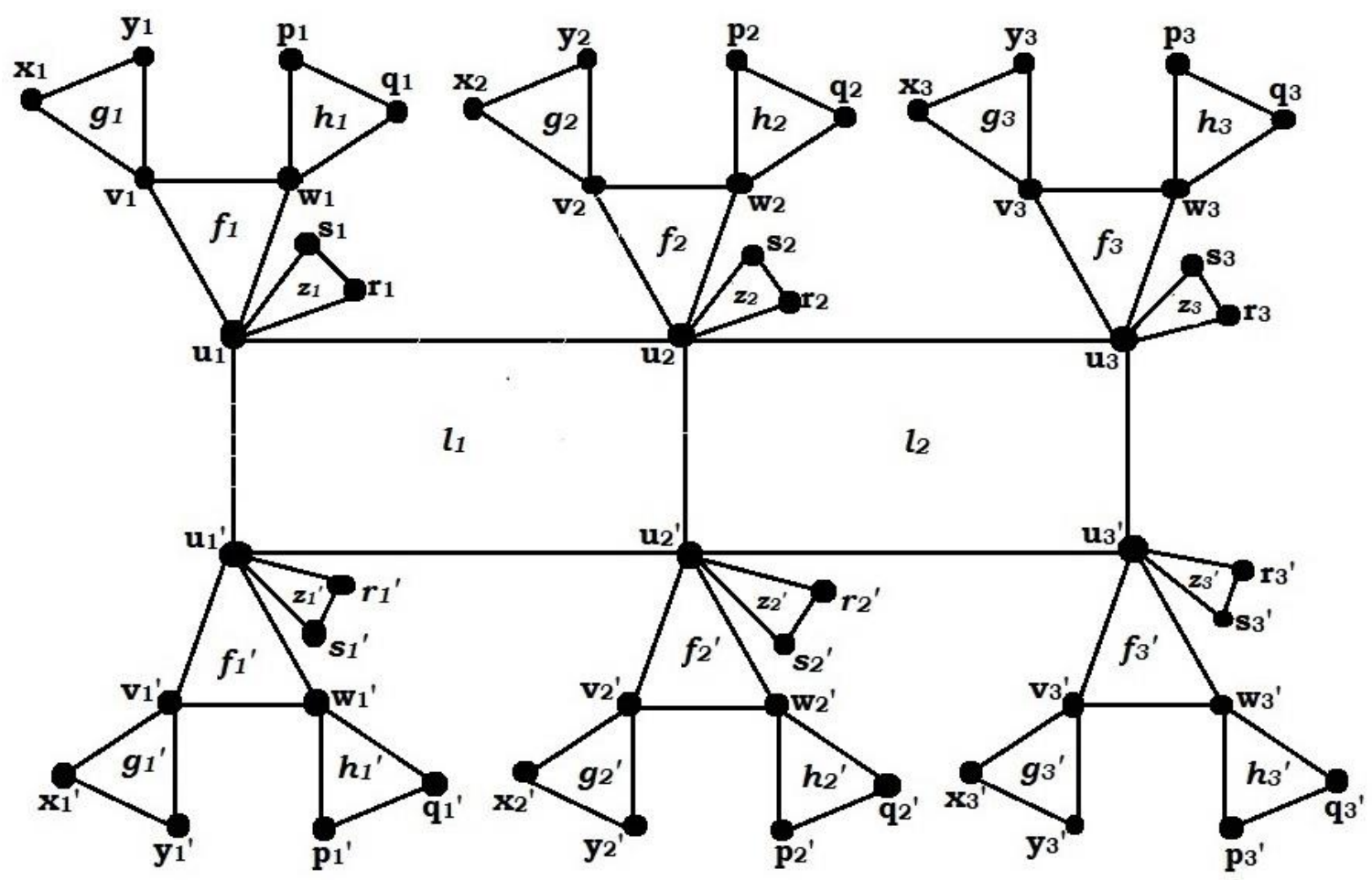

Figure 2. $\mathrm{DD}_{\mathrm{vv}}\left(L_{3}\right)$

The following are the face magic labeling of types $(1,0,1),(1,1,0)$ and $(0,1,1)$.

Type (i) : (1,0,1) - Face Magic

Define a mapping $\alpha_{1}: \mathrm{V}^{\prime} \mathrm{UF}^{\prime} \rightarrow\{1,2,3, \ldots, 27 \mathrm{n}-1\}$ as follows.

$$
\begin{gathered}
\alpha_{1}\left(u_{k}\right)=k, \\
\alpha_{1}\left(x_{k}\right)=16 n+k, \\
\alpha_{1}\left(q_{k}\right)=8 n+k, \\
\alpha_{1}\left(f_{k}\right)=26 n+1-k,
\end{gathered}
$$

$$
\text { For } 1 \leq k \leq n \text {, }
$$

$$
\alpha_{1}\left(v_{k}\right)=4 n+k, \quad \alpha_{1}\left(w_{k}\right)=4 n+1-k,
$$$$
\alpha_{1}\left(y_{k}\right)=8 n+1-k
$$$$
\alpha_{1}\left(r_{k}\right)=12 n+1-k \text {, }
$$$$
\alpha_{1}\left(g_{k}\right)=20 n+1-k \text {, }
$$$$
\alpha_{1}\left(z_{k}\right)=24 n+1-k \text {. }
$$ 
For $1 \leq k \leq n-2, \quad \alpha_{1}\left(l_{k}\right)=27 n-1-k, \quad \alpha_{1}\left(l_{n-1}\right)=27 n-1$.

Case(i): $\mathrm{n} \equiv 1(\bmod 2)$

For $1 \leq k \leq \frac{n+1}{2}$

$$
\begin{aligned}
& \alpha_{1}\left(u_{2 k-1}^{\prime}\right)=2 n+1-k, \\
& \alpha_{1}\left(x_{2 k-1}^{\prime}\right)=10 n+1-k, \\
& \alpha_{1}\left(q_{2 k-1}^{\prime}\right)=18 n+1-k, \\
& \alpha_{1}\left(f_{2 k-1}^{\prime}\right)=24 n+k,
\end{aligned}
$$

$$
\begin{aligned}
& \alpha_{1}\left(v_{2 k-1}^{\prime}\right)=2 n+k, \\
& \alpha_{1}\left(y_{2 k-1}^{\prime}\right)=14 n+k, \\
& \alpha_{1}\left(r_{2 k-1}^{\prime}\right)=14 n+1-k, \\
& \alpha_{1}\left(g_{2 k-1}^{\prime}\right)=22 n+1-k, \\
& \alpha_{1}\left(z_{2 k-1}^{\prime}\right)=22 n+k .
\end{aligned}
$$

$$
\begin{gathered}
\alpha_{1}\left(w_{2 k-1}^{\prime}\right)=6 n+1-k \\
\alpha_{1}\left(p_{2 k-1}^{\prime}\right)=6 n+k \\
\alpha_{1}\left(s_{2 k-1}^{\prime}\right)=10 n+k \\
\alpha_{1}\left(h_{2 k-1}^{\prime}\right)=18 n+k
\end{gathered}
$$

For $1 \leq k \leq \frac{n-1}{2}$

$$
\begin{aligned}
& \alpha_{1}\left(u_{2 k}^{\prime}\right)=\frac{3 n+1}{2}-k, \\
& \alpha_{1}\left(x_{2 k}^{\prime}\right)=\frac{19 n+1}{2}-k, \\
& \alpha_{1}\left(q_{2 k}^{\prime}\right)=\frac{35 n+1}{2}-k, \\
& \alpha_{1}\left(f_{2 k}^{\prime}\right)=\frac{49 n+1}{2}+k, \\
& \alpha_{1}\left(z_{2 k}^{\prime}\right)=\frac{45 n+1}{2}+k .
\end{aligned}
$$

$$
\begin{aligned}
& \alpha_{1}\left(v_{2 k}^{\prime}\right)=\frac{5 n+1}{2}+k, \\
& \alpha_{1}\left(y_{2 k}^{\prime}\right)=\frac{29 n+1}{2}+k, \\
& \alpha_{1}\left(r_{2 k}^{\prime}\right)=\frac{27 n+1}{2}-k, \\
& \alpha_{1}\left(g_{2 k}^{\prime}\right)=\frac{43 n+1}{2}-k,
\end{aligned}
$$

$$
\begin{aligned}
& \alpha_{1}\left(w_{2 k}^{\prime}\right)=\frac{11 n+1}{2}-k, \\
& \alpha_{1}\left(p_{2 k}^{\prime}\right)=\frac{13 n+1}{2}+k, \\
& \alpha_{1}\left(s_{2 k}^{\prime}\right)=\frac{21 n+1}{2}+k, \\
& \alpha_{1}\left(h_{2 k}^{\prime}\right)=\frac{37 n+1}{2}+k,
\end{aligned}
$$

Case(ii): $\mathrm{n} \equiv 0(\bmod 2)$

For $1 \leq k \leq \frac{n}{2}$

$$
\begin{aligned}
& \begin{array}{lcrl}
\alpha_{1}\left(u_{2 k-1}^{\prime}\right)=\frac{3 n}{2}+1-k, & \alpha_{1}\left(v_{2 k-1}^{\prime}\right)=\frac{5 n}{2}+k, & \alpha_{1}\left(w_{2 k-1}^{\prime}\right)=\frac{11 n}{2}+1-k, \\
\alpha_{1}\left(x_{2 k-1}^{\prime}\right)=\frac{19 n}{2}+1-k, & \alpha_{1}\left(y_{2 k-1}^{\prime}\right)=\frac{29 n}{2}+k, & \alpha_{1}\left(p_{2 k-1}^{\prime}\right)=\frac{13 n}{2}+k
\end{array} \\
& \alpha_{1}\left(x_{2 k-1}^{\prime}\right)=\frac{19 n}{2}+1-k, \quad \alpha_{1}\left(y_{2 k-1}^{\prime}\right)=\frac{29 n}{2}+k, \quad \alpha_{1}\left(p_{2 k-1}^{\prime}\right)=\frac{13 n}{2}+k, \\
& \alpha_{1}\left(q_{2 k-1}^{\prime}\right)=\frac{35 n}{2}+1-k, \quad \alpha_{1}\left(r_{2 k-1}^{\prime}\right)=\frac{27 n}{2}+1-k, \quad \alpha_{1}\left(s_{2 k-1}^{\prime}\right)=\frac{21 n}{2}+k, \\
& \alpha_{1}\left(f_{2 k-1}^{\prime}\right)=\frac{49 n}{2}+k \quad, \quad \alpha_{1}\left(g_{2 k-1}^{\prime}\right)=\frac{43 n}{2}+1-k, \quad \alpha_{1}\left(h_{2 k-1}^{\prime}\right)=\frac{37 n}{2}+k, \\
& \alpha_{1}\left(z_{2 k-1}^{\prime}\right)=\frac{45 n}{2}+k, \quad \alpha_{1}\left(z_{2 k}^{\prime}\right)=22 n+k \text {. } \\
& \alpha_{1}\left(u_{2 k}^{\prime}\right)=2 n+1-k, \\
& \alpha_{1}\left(x_{2 k}^{\prime}\right)=10 n+1-k \text {, } \\
& \alpha_{1}\left(q^{\prime}{ }_{2 k}\right)=18 n+1-k \text {, } \\
& \alpha_{1}\left(f^{\prime}{ }_{2 k}\right)=24 n+k \text {, } \\
& \alpha_{1}\left(v^{\prime}{ }_{2 k}\right)=2 n+k, \\
& \alpha_{1}\left(y_{2 k}^{\prime}\right)=14 n+k \text {, } \\
& \alpha_{1}\left(r^{\prime}{ }_{2 k}\right)=14 n+1-k \text {, } \\
& \alpha_{1}\left(g^{\prime}{ }_{2 k}\right)=22 n+1-k \text {, } \\
& \alpha_{1}\left(w_{2 k}^{\prime}\right)=6 n+1-k, \\
& \alpha_{1}\left(p^{\prime}{ }_{2 k}\right)=6 n+k \text {, } \\
& \alpha_{1}\left(s^{\prime}{ }_{2 k}\right)=10 n+k \text {, } \\
& \alpha_{1}\left(h^{\prime}{ }_{2 k}\right)=18 n+k \text {. }
\end{aligned}
$$

Thus, the above labeling pattern gives the weight of all 3-sided and 4-sided faces as follows, For $1 \leq k \leq n$,

The weight of all 3-sided faces is given by,

$\mathrm{w}_{1}\left(g_{k}\right)=\alpha_{1}\left(v_{k}\right)+\alpha_{1}\left(x_{k}\right)+\alpha_{1}\left(y_{k}\right)+\alpha_{1}\left(g_{k}\right)=48 n+2=k_{1}$

Similarly, $\mathrm{w}_{1}\left(g_{k}^{\prime}\right)=\mathrm{w}_{1}\left(h_{k}\right)=\mathrm{w}_{1}\left(h_{k}^{\prime}\right)=\mathrm{w}_{1}\left(z_{k}\right)=\mathrm{w}_{1}\left(z_{k}^{\prime}\right)=48 n+2=k_{1}$

$$
\mathrm{w}_{1}\left(f_{k}\right)=\alpha_{1}\left(u_{k}\right)+\alpha_{1}\left(v_{k}\right)+\alpha_{1}\left(w_{k}\right)+\alpha_{1}\left(f_{k}\right)=34 n+2=k_{2}
$$

Similarly, $\mathrm{w}_{1}\left(f_{k}^{\prime}\right)=34 n+2=k_{2}$

The weight of all 4 -sided faces,

For $k=n-l, \quad \mathrm{w}_{2}\left(l_{k}\right)=\alpha_{1}\left(u_{k}\right)+\alpha_{1}\left(u_{k+1}\right)+\alpha_{1}\left(u_{k}^{\prime}\right)+\alpha_{1}\left(u_{k+1}^{\prime}\right)+\alpha_{1}\left(l_{k}\right)=k_{l}$

$$
k_{1}=\left\{\begin{array}{l}
\frac{63 n-1}{2} ; n \text { is odd } \\
\frac{63 n}{2} ; n \text { is even }
\end{array}\right.
$$

For $1 \leq k \leq n-2, \mathrm{w}_{2}\left(l_{k}\right)=\alpha_{1}\left(u_{k}\right)+\alpha_{1}\left(u_{k+1}\right)+\alpha_{1}\left(u_{k}^{\prime}\right)+\alpha_{1}\left(u_{k+1}^{\prime}\right)+\alpha_{1}\left(l_{k}\right)=k_{2}$

$$
k_{2}= \begin{cases}\frac{61 n+1}{2} ; & n \text { is odd } \\ \frac{61 n+2}{2} ; & n \text { is even }\end{cases}
$$


Type(ii): $(1,1,0)$ - Face Magic

Define a mapping $\alpha_{2}: \mathrm{V}^{\prime} \cup E^{\prime} \rightarrow\{1,2,3, \ldots, 45 n-2\}$ as follows.

For $1 \leq k \leq n$,

$$
\begin{array}{llr}
\alpha_{2}\left(u_{k}\right)=k, & \alpha_{2}\left(v_{k}\right)=4 n+k, & \alpha_{2}\left(w_{k}\right)=4 n+1-k, \\
\alpha_{2}\left(x_{k}\right)=16 n+k, & \alpha_{2}\left(y_{k}\right)=8 n+1-k, & \alpha_{2}\left(p_{k}\right)=16 n+1-k, \\
\alpha_{2}\left(q_{k}\right)=8 n+k, & \alpha_{2}\left(r_{k}\right)=12 n+1-k, & \alpha_{2}\left(s_{k}\right)=12 n+k, \\
\alpha_{2}\left(u_{k} v_{k}\right)=38 n+k, & \alpha_{2}\left(u_{k} w_{k}\right)=42 n+1-k, & \alpha_{2}\left(v_{k} w_{k}\right)=38 n+1-k, \\
\alpha_{2}\left(v_{k} x_{k}\right)=32 n+1-k, & \alpha_{2}\left(v_{k} y_{k}\right)=28 n+k, & \alpha_{2}\left(x_{k} y_{k}\right)=20 n+1-k, \\
\alpha_{2}\left(w_{k} p_{k}\right)=32 n+k, & \alpha_{2}\left(w_{k} q_{k}\right)=28 n+1-k, & \alpha_{2}\left(p_{k} q_{k}\right)=20 n+k, \\
\alpha_{2}\left(u_{k} r_{k}\right)=24 n+k, & \alpha_{2}\left(u_{k} s_{k}\right)=36 n+1-k, & \alpha_{2}\left(r_{k} s_{k}\right)=24 n+1-k .
\end{array}
$$

For $1 \leq k \leq n-1, \quad \alpha_{2}\left(u_{k} u_{k+1}\right)=42 n+k$, $\alpha_{2}\left(u_{n} u_{n}^{\prime}\right)=45 n-2$.

$$
\alpha_{2}\left(u_{k}^{\prime} u_{k+1}^{\prime}\right)=44 n-1-k
$$

Case(i): $\mathrm{n} \equiv 1(\bmod 2)$

For $1 \leq k \leq \frac{n+1}{2}$

$$
\begin{array}{ccc}
\alpha_{2}\left(u_{2 k-1}^{\prime}\right)=2 n+1-k, & \alpha_{2}\left(v_{2 k-1}^{\prime}\right)=2 n+k, & \alpha_{2}\left(w_{2 k-1}^{\prime}\right)=6 n+1-k, \\
\alpha_{2}\left(x_{2 k-1}^{\prime}\right)=10 n+1-k, & \alpha_{2}\left(y_{2 k-1}^{\prime}\right)=14 n+k, & \alpha_{2}\left(p_{2 k-1}^{\prime}\right)=6 n+k, \\
\alpha_{2}\left(q_{2 k-1}^{\prime}\right)=18 n+1-k, & \alpha_{2}\left(r_{2 k-1}^{\prime}\right)=14 n+1-k, & \alpha_{2}\left(s_{2 k-1}^{\prime}\right)=10 n+k, \\
\alpha_{2}\left(u_{2 k-1}^{\prime} v_{2 k-1}^{\prime}\right)=40 n+k, & \alpha_{2}\left(u_{2 k-1}^{\prime} w_{2 k-1}^{\prime}\right)=40 n+1-k, & \alpha_{2}\left(v_{2 k-1}^{\prime} w_{2 k-1}^{\prime}\right)=36 n+k, \\
\alpha_{2}\left(v_{2 k-1}^{\prime} x_{2 k-1}^{\prime}\right)=26 n+k, & \alpha_{2}\left(v_{2 k-1}^{\prime} y_{2 k-1}^{\prime}\right)=34 n+1-k, & \alpha_{2}\left(x_{2 k-1}^{\prime} y_{2 k-1}^{\prime}\right)=22 n+1-k, \\
\alpha_{2}\left(w_{2 k-1}^{\prime} p_{2 k-1}^{\prime}\right)=30 n+1-k, & \alpha_{2}\left(w_{2 k-1}^{\prime} q_{2 k-1}^{\prime}\right)=30 n+k, & \alpha_{2}\left(p_{2 k-1}^{\prime} q_{2 k-1}^{\prime}\right)=18 n+k, \\
\alpha_{2}\left(u_{2 k-1}^{\prime} r_{2 k-1}^{\prime}\right)=26 n+1-k, & \alpha_{2}\left(u_{2 k-1}^{\prime} s_{2 k-1}^{\prime}\right)=34 n+k, & \alpha_{2}\left(r_{2 k-1}^{\prime} s_{2 k-1}^{\prime}\right)=22 n+k .
\end{array}
$$

For $1 \leq k \leq \frac{n-1}{2}, \quad \alpha_{2}\left(u_{2 k-1} u_{2 k-1}^{\prime}\right)=45 n-2-k$

For $1 \leq k \leq \frac{n-1}{2}$

$$
\begin{array}{rlrl}
\alpha_{2}\left(u_{2 k}^{\prime}\right)=\frac{3 n+1}{2}-k, & \alpha_{2}\left(v_{2 k}^{\prime}\right)=\frac{5 n+1}{2}+k, & & \alpha_{2}\left(w_{2 k}^{\prime}\right)=\frac{11 n+1}{2}-k, \\
\alpha_{2}\left(x_{2 k}^{\prime}\right)=\frac{19 n+1}{2}-k, & \alpha_{2}\left(y_{2 k}^{\prime}\right)=\frac{29 n+1}{2}+k, & \alpha_{2}\left(p_{2 k}^{\prime}\right)=\frac{13 n+1}{2}+k, \\
\alpha_{2}\left(q_{2 k}^{\prime}\right)=\frac{35 n+1}{2}-k, & \alpha_{2}\left(r_{2 k}^{\prime}\right)=\frac{27 n+1}{2}-k, & & \alpha_{2}\left(s_{2 k}^{\prime}\right)=\frac{21 n+1}{2}+k, \\
\alpha_{2}\left(u_{2 k}^{\prime} v_{2 k}^{\prime}\right)=\frac{81 n+1}{2}+k, & \alpha_{2}\left(u_{2 k}^{\prime}{w^{\prime}}_{2 k}\right)=\frac{79 n+1}{2}-k, & \alpha_{2}\left(v_{2 k}^{\prime} w_{2 k}^{\prime}\right)=\frac{73 n+1}{2}+k, \\
\alpha_{2}\left(v_{2 k}^{\prime} x_{2 k}^{\prime}\right)=\frac{53 n+1}{2}+k, & \alpha_{2}\left(v_{2 k}^{\prime} y_{2 k}^{\prime}\right)=\frac{67 n+1}{2}-k, & \alpha_{2}\left(x_{2 k}^{\prime} y_{2 k}^{\prime}\right)=\frac{43 n+1}{2}-k, \\
\alpha_{2}\left(w_{2 k}^{\prime} p_{2 k}^{\prime}\right)=\frac{59 n+1}{2}-k, & \alpha_{2}\left(w_{2 k}^{\prime} q_{2 k}^{\prime}\right)=\frac{61 n+1}{2}+k, & \alpha_{2}\left(p_{2 k}^{\prime} q_{2 k}^{\prime}\right)=\frac{37 n+1}{2}+k, \\
\alpha_{2}\left(u_{2 k}^{\prime} r_{2 k}^{\prime}\right)=\frac{51 n+1}{2}-k, & \alpha_{2}\left(r_{2 k}^{\prime} s_{2 k}^{\prime}\right)=\frac{45 n+}{2}+k, & \alpha_{2}\left(u_{2 k}^{\prime} s_{2 k}^{\prime}\right)=\frac{69 n+1}{2}+k .
\end{array}
$$

Case(ii): $\mathrm{n} \equiv 0(\bmod 2)$

For $1 \leq k \leq \frac{n}{2}$

$$
\begin{aligned}
& \alpha_{2}\left(u_{2 k-1}^{\prime}\right)=\frac{3 n}{2}+1-k, \quad \alpha_{2}\left(v_{2 k-1}^{\prime}\right)=\frac{5 n}{2}+k, \quad \alpha_{2}\left(w_{2 k-1}^{\prime}\right)=\frac{11 n}{2}+1-k, \\
& \alpha_{2}\left(x_{2 k-1}^{\prime}\right)=\frac{19 n}{2}+1-k, \quad \alpha_{2}\left(y_{2 k-1}^{\prime}\right)=\frac{29 n}{2}+k, \quad \alpha_{2}\left(p_{2 k-1}^{\prime}\right)=\frac{13 n}{2}+k, \\
& \alpha_{2}\left(q_{2 k-1}^{\prime}\right)=\frac{35 n}{2}+1-k, \quad \alpha_{2}\left(r_{2 k-1}^{\prime}\right)=\frac{27 n}{2}+1-k, \quad \alpha_{2}\left(s_{2 k-1}^{\prime}\right)=\frac{21 n}{2}+k, \\
& \alpha_{2}\left(u_{2 k-1}^{\prime} v_{2 k-1}^{\prime}\right)=\frac{81 n}{2}+k, \quad \alpha_{2}\left(u_{2 k-1}^{\prime} w_{2 k-1}^{\prime}\right)=\frac{79 n+2}{2}-k \quad, \quad \alpha_{2}\left(v_{2 k-1}^{\prime} w^{\prime}{ }_{2 k-1}\right)=\frac{73 n}{2}+k \text {, } \\
& \alpha_{2}\left(v_{2 k-1}^{\prime} x_{2 k-1}^{\prime}\right)=\frac{53 n}{2}+k, \alpha_{2}\left(v_{2 k-1}^{\prime} y_{2 k-1}^{\prime}\right)=\frac{67 n+2}{2}-k, \quad \alpha_{2}\left(x_{2 k-1}^{\prime} y_{2 k-1}^{\prime}\right)=\frac{43 n+2}{2}-k \text {, } \\
& \alpha_{2}\left(w_{2 k-1}^{\prime} p_{2 k-1}^{\prime}\right)=\frac{59 n+2}{2}-k, \quad \alpha_{2}\left(w_{2 k-1}^{\prime} q_{2 k-1}^{\prime}\right)=\frac{61 n}{2}+k, \quad \alpha_{2}\left(p_{2 k-1}^{\prime} q_{2 k-1}^{\prime}\right)=\frac{37 n}{2}+k \text {, }
\end{aligned}
$$




$$
\begin{aligned}
& \alpha_{2}\left(u_{2 k-1}^{\prime} r_{2 k-1}^{\prime}\right)=\frac{51 n+2}{2}-k, \quad \alpha_{2}\left(r_{2 k-1}^{\prime} s_{2 k-1}^{\prime}\right)=\frac{45 n}{2}+k, \quad \alpha_{2}\left(u_{2 k-1}^{\prime} s_{2 k-1}^{\prime}\right)=\frac{69 n}{2}+k \text {. } \\
& \alpha_{1}\left(u_{2 k}^{\prime}\right)=2 n+1-k \text {, } \\
& \alpha_{2}\left(u_{2 k-1} u_{2 k-1}^{\prime}\right)=\frac{89 n-2}{2}-k \text {. } \\
& \alpha_{1}\left(x_{2 k}^{\prime}\right)=10 n+1-k \text {, } \\
& \alpha_{1}\left(v_{2 k}^{\prime}\right)=2 n+k, \quad \alpha_{1}\left(w_{2 k}^{\prime}\right)=6 n+1-k, \\
& \alpha_{1}\left(q_{2 k}^{\prime}\right)=18 n+1-k \\
& \alpha_{2}\left(u_{2 k}^{\prime} v_{2 k}^{\prime}\right)=40 n+k \text {, } \\
& \alpha_{2}\left(v_{2 k}^{\prime} x_{2 k}^{\prime}\right)=26 n+k \text {, } \\
& \alpha_{2}\left(w_{2 k}^{\prime} p_{2 k}^{\prime}\right)=30 n+1-k \text {, } \\
& \alpha_{2}\left(u_{2 k}^{\prime} r_{2 k}^{\prime}\right)=26 n+1-k \text {, } \\
& \alpha_{1}\left(y_{2 k}^{\prime}\right)=14 n+k \text {, } \\
& \alpha_{1}\left(p_{2 k}^{\prime}\right)=6 n+k \text {, } \\
& \alpha_{1}\left(r_{2 k}^{\prime}\right)=14 n+1-k \text {, } \\
& \alpha_{1}\left(s^{\prime}{ }_{2 k}\right)=10 n+k \text {, } \\
& \alpha_{2}\left(u_{2 k}^{\prime} w^{\prime}{ }_{2 k}\right)=40 n+1-k, \alpha_{2}\left(v_{2 k}^{\prime} w_{2 k}^{\prime}\right)=36 n+k \text {, } \\
& \alpha_{2}\left(v_{2 k}^{\prime} y_{2 k}^{\prime}\right)=34 n+1-k, \quad \alpha_{2}\left(x_{2 k}^{\prime} y_{2 k}^{\prime}\right)=22 n+1-k, \\
& \alpha_{2}\left(u_{2 k}^{\prime} s_{2 k}^{\prime}\right)=34 n+k \text {. }
\end{aligned}
$$

Thus, the above labeling pattern gives the weight of all 3-sided and 4-sided faces as follows,

For $1 \leq k \leq n$,

The weight of all 3-sided faces is given by,

$$
\begin{aligned}
& \mathrm{w}_{1}\left(g_{k}\right)=\alpha_{2}\left(v_{k}\right)+\alpha_{2}\left(x_{k}\right)+\alpha_{2}\left(y_{k}\right)+\alpha_{2}\left(v_{k} x_{k}\right)+\alpha_{2}\left(x_{k} y_{k}\right)+\alpha_{2}\left(v_{k} y_{k}\right)=108 n+3=k_{1} \\
& \text { Similarly, } \mathrm{w}_{1}\left(g_{k}^{\prime}\right)=\mathrm{w}_{1}\left(h_{k}\right)=\mathrm{w}_{1}\left(h_{k}^{\prime}\right)=\mathrm{w}_{1}\left(z_{k}\right)=\mathrm{w}_{1}\left(z_{k}^{\prime}\right)=108 n+3=k_{1} \\
& \mathrm{w}_{1}\left(f_{k}\right)=\alpha_{2}\left(u_{k}\right)+\alpha_{2}\left(v_{k}\right)+\alpha_{2}\left(w_{k}\right)+\alpha_{2}\left(u_{k} v_{k}\right)+\alpha_{2}\left(u_{k} w_{k}\right)+\alpha_{2}\left(v_{k} w_{k}\right)=126 n+3=k_{2} \\
& \text { Similarly, } \mathrm{w}_{1}\left(f_{k}^{\prime}\right)=126 n+3=k_{2} \\
& \text { The weight of all 4-sided faces, } \\
& \text { For } k=n-1, \mathrm{w}_{2}\left(l_{k}\right)=\alpha_{2}\left(u_{k}\right)+\alpha_{2}\left(u_{k+1}\right)+\alpha_{2}\left(u_{k}^{\prime}\right)+\alpha_{2}\left(u_{k+1}^{\prime}\right)+\alpha_{2}\left(u_{k} u_{k+1}\right) \\
& k_{1}= \begin{cases}\frac{359 n-7}{2} ; & n \text { is odd } \\
\frac{359 n-6}{2} ; & n \text { is even }\end{cases}
\end{aligned}
$$

For $1 \leq k \leq n-2, \mathrm{w}_{2}\left(l_{k}\right)=\alpha_{2}\left(u_{k}\right)+\alpha_{2}\left(u_{k+1}\right)+\alpha_{2}\left(u_{k}^{\prime}\right)+\alpha_{2}\left(u_{k+1}^{\prime}\right)+\alpha_{2}\left(u_{k} u_{k+1}\right)$

$$
\begin{gathered}
+\alpha_{2}\left(u_{k}^{\prime} u_{k+1}^{\prime}\right)+\alpha_{2}\left(u_{k} u_{k}^{\prime}\right)+\alpha_{2}\left(u_{k+1} u_{k+1}^{\prime}\right)=k_{2} \\
k_{2}=\left\{\begin{array}{cc}
178 n-4 ; & n \text { is odd } \\
179 n-3 ; & n \text { is even }
\end{array}\right.
\end{gathered}
$$

Type(iii): $(0,1,1)$

Define a function $\alpha_{3}: E^{\prime} \cup F^{\prime} \rightarrow\{1,2,3, \ldots, 36 n-3\}$ as follows,

For $1 \leq k \leq n-1, \quad \alpha_{3}\left(u_{k} u_{k+1}\right)=k, \quad \alpha_{3}\left(u_{k}^{\prime} u_{k+1}^{\prime}\right)=2 n-1-k$

For $1 \leq k \leq n$,

$$
\begin{array}{lcc}
\alpha_{3}\left(u_{k} v_{k}\right)=7 n-2+k, & \alpha_{3}\left(u_{k} w_{k}\right)=7 n-1-k, & \alpha_{3}\left(v_{k} w_{k}\right)=3 n-2+k, \\
\alpha_{3}\left(v_{k} x_{k}\right)=23 n-2+k, & \alpha_{3}\left(v_{k} y_{k}\right)=19 n-1-k, & \alpha_{3}\left(x_{k} y_{k}\right)=11 n-2+k, \\
\alpha_{3}\left(w_{k} p_{k}\right)=23 n-1-k, & \alpha_{3}\left(w_{k} q_{k}\right)=19 n-2+k & \alpha_{3}\left(p_{k} q_{k}\right)=11 n-1-k, \\
\alpha_{3}\left(u_{k} r_{k}\right)=27 n-1-k, & \alpha_{3}\left(u_{k} s_{k}\right)=15 n-2+k, & \alpha_{3}\left(r_{k} s_{k}\right)=15 n-1-k . \\
& & \\
\alpha_{3}\left(u_{k}^{\prime} v_{k}^{\prime}\right)=5 n-2+k, & \alpha_{3}\left(u_{k}^{\prime} w_{k}^{\prime}\right)=9 n-1-k, & \alpha_{3}\left(v_{k}^{\prime} w_{k}^{\prime}\right)=5 n-1-k, \\
\alpha_{3}\left(v_{k}^{\prime} x_{k}^{\prime}\right)=21 n-1-k, & \alpha_{3}\left(v_{k}^{\prime} y_{k}^{\prime}\right)=21 n-2+k, & \alpha_{3}\left(x_{k}^{\prime} y_{k}^{\prime}\right)=9 n-2+k, \\
\alpha_{3}\left(w_{k}^{\prime} p_{k}^{\prime}\right)=17 n-2+k, & \alpha_{3}\left(w_{k}^{\prime} q_{k}^{\prime}\right)=25 n-1-k, & \alpha_{3}\left(p_{k}^{\prime} q_{k}^{\prime}\right)=13 n-1-k, \\
\alpha_{3}\left(u_{k}^{\prime} r_{k}^{\prime}\right)=17 n-1-k, & \alpha_{3}\left(u_{k}^{\prime} s_{k}^{\prime}\right)=25 n-2+k, & \alpha_{3}\left(r_{k}^{\prime} s_{k}^{\prime}\right)=13 n-2+k, \\
& & \\
\alpha_{3}\left(f_{k}\right)=35 n-1-k, & \alpha_{3}\left(f_{k}^{\prime}\right)=33 n-2+k, & \alpha_{3}\left(g_{k}\right)=31 n-1-k, \\
\alpha_{3}\left(g_{k}^{\prime}\right)=33 n-1-k, & \alpha_{3}\left(h_{k}\right)=31 n-2+k, & \alpha_{3}\left(h_{k}^{\prime}\right)=29 n-2+k, \\
\alpha_{3}\left(z_{k}\right)=27 n-2+k, & \alpha_{3}\left(z_{k}^{\prime}\right)=29 n-1-k, & \alpha_{3}\left(l_{n-1}\right)=36 n-3 .
\end{array}
$$

For $1 \leq k \leq n-2, \quad \alpha_{3}\left(l_{k}\right)=36 n-3-k$

Case(i): $n \equiv 1(\bmod 2)$

For $1 \leq k \leq \frac{n+1}{2}, \quad \alpha_{3}\left(u_{2 k-1} u_{2 k-1}^{\prime}\right)=2 n+\frac{n-5}{2}+k$, 
For $1 \leq k \leq \frac{n-1}{2}, \quad \alpha_{3}\left(u_{2 k} u_{2 k}^{\prime}\right)=2 n-2+k$,

Case(ii): $\mathrm{n} \equiv 0(\bmod 2)$

For $1 \leq k \leq \frac{n}{2}, \quad \alpha_{3}\left(u_{2 k-1} u_{2 k-1}^{\prime}\right)=2 n+\frac{n-4}{2}+k, \quad \alpha_{3}\left(u_{2 k} u_{2 k}^{\prime}\right)=2 n-2+k$,

The following are the weights of all 3-sided and 4-sided faces of a ladder graph,

For $1 \leq k \leq n$,

The weight of all 3-sided faces is given by,

$\mathrm{w}_{1}\left(g_{k}\right)=\alpha_{3}\left(v_{k} x_{k}\right)+\alpha_{3}\left(x_{k} y_{k}\right)+\alpha_{3}\left(v_{k} y_{k}\right)+\alpha_{3}\left(g_{k}\right)=84 n-6=k_{l}$

Similarly, $\mathrm{w}_{1}\left(g_{k}^{\prime}\right)=\mathrm{w}_{1}\left(h_{k}\right)=\mathrm{w}_{1}\left(h_{k}^{\prime}\right)=\mathrm{w}_{1}\left(z_{k}\right)=\mathrm{w}_{1}\left(z_{k}^{\prime}\right)=84 n-6=k_{l}$

Also, $\mathrm{w}_{1}\left(f_{k}\right)=\alpha_{3}\left(u_{k} v_{k}\right)+\alpha_{3}\left(u_{k} w_{k}\right)+\alpha_{3}\left(v_{k} w_{k}\right)+\alpha_{3}\left(f_{k}\right)=52 n-6=k_{2}$

Similarly, $\mathrm{w}_{1}\left(f_{k}^{\prime}\right)=52 n-6=k_{2}$

The weight of all 4-sided faces,

For $k=n-1$,

$\mathrm{w}_{2}\left(l_{k}\right)=\alpha_{3}\left(u_{k} u_{k+1}\right)+\alpha_{3}\left(u_{k}^{\prime} u_{k+1}^{\prime}\right)+\alpha_{3}\left(u_{k} u_{k}^{\prime}\right)+\alpha_{3}\left(u_{k+1} u_{k+1}^{\prime}\right)+\alpha_{3}\left(l_{k}\right)=k_{1}$

$$
k_{1}= \begin{cases}\frac{87 n-17}{2} ; & n \text { is odd } \\ \frac{87 n-16}{2} ; & n \text { is even }\end{cases}
$$

For $1 \leq k \leq n-2$,

$\mathrm{w}_{2}\left(l_{k}\right)=\alpha_{3}\left(u_{k} u_{k+1}\right)+\alpha_{3}\left(u_{k}^{\prime} u_{k+1}^{\prime}\right)+\alpha_{3}\left(u_{k} u_{k}^{\prime}\right)+\alpha_{3}\left(u_{k+1} u_{k+1}^{\prime}\right)+\alpha_{3}\left(l_{k}\right)=k_{2}$

$$
k_{2}= \begin{cases}\frac{85 n-15}{2} ; & n \text { is odd } \\ \frac{85 n-14}{2} ; & n \text { is even }\end{cases}
$$

Hence the graph $\mathrm{DD}_{\mathrm{Vv}}\left(\mathrm{L}_{\mathrm{n}}\right), \mathrm{n} \geq 3$ of types $(1,0,1),(1,1,0)$ and $(0,1,1)$ is face bimagic.

Theorem 2.2. If $G$ is $(1,0,1)$ face bimagic except for 3 -sided faces then $\operatorname{DD}_{V v}(G)$ is $(1,0,1)$ face bimagic.

\section{Proof:}

By assumption the graph $G(V, E, F)$ with $p$ vertices, $e$ edges and $g$ faces is face bimagic. Then there exists a mapping $\lambda: \mathrm{V} \cup \mathrm{F} \rightarrow\{1,2,3, \ldots, \mathrm{p}+\mathrm{g}\}$ such that the weight of each face is either $\mathrm{k}_{1}$ or $\mathrm{k}_{2}$ constant. The vertex set and the face set of $\mathrm{G}$ are,

$\mathrm{V}=\left\{v_{k}, 1 \leq k \leq p\right\}$ and $\mathrm{F}=\left\{g_{k}=\mathrm{p}+\mathrm{x}, 1 \leq x \leq g\right\}$.

Let $G^{\prime}\left(V^{\prime}, E^{\prime}, F^{\prime}\right)$ denotes the double duplication of all vertices by edges of $G$ with,

$$
\begin{gathered}
\mathrm{V}^{\prime}=\mathrm{V} \cup\left\{u_{k}, w_{k}, v_{k}^{\prime}, u_{k}^{\prime}, w_{k}^{\prime}, v_{k}^{\prime \prime}, u_{k}^{\prime \prime}, w_{k}^{\prime \prime}: 1 \leq k \leq p\right\} \\
\mathrm{E}^{\prime}=\mathrm{E} \cup\left\{v_{k} u_{k}, u_{k} w_{k}, v_{k} w_{k}, v_{k} v_{k}^{\prime}, v_{k} v_{k}^{\prime \prime}, v_{k}^{\prime} u_{k}^{\prime}, v_{k}^{\prime} w_{k}^{\prime}, v_{k}^{\prime \prime} u_{k}^{\prime \prime}, v_{k}^{\prime \prime} w_{k}^{\prime \prime}, u_{k}^{\prime \prime} w_{k}^{\prime \prime}: 1 \leq k \leq p\right\} \\
\mathrm{F}^{\prime}=\mathrm{F} \cup\left\{f_{k}: v_{k} v_{k}^{\prime} v_{k}^{\prime \prime}, 1 \leq k \leq p\right\} \bigcup\left\{f_{k}^{\prime}: u_{k} v_{k} w_{k}, 1 \leq k \leq p\right\} \bigcup\left\{f_{k}^{\prime \prime}: v_{k}^{\prime} u_{k}^{\prime} w_{k}^{\prime}, 1 \leq k \leq p\right\} \\
\bigcup\left\{f_{k}^{\prime \prime \prime}: v_{k}^{\prime \prime} u_{k}^{\prime \prime} w_{k}^{\prime \prime}, 1 \leq k \leq p\right\} .
\end{gathered}
$$

Consider a mapping $\lambda^{\prime}: V^{\prime} \cup F^{\prime} \rightarrow\{1,2,3, \ldots, 13 p+g\}$

To prove $G^{\prime}$ is face bimagic it is enough to prove $(1,0,1)$ face bimagic for newly added vertices and edges.

For $1 \leq k \leq p$,

$$
\begin{array}{lrrl}
\lambda^{\prime}\left(v_{k}\right)=\lambda\left(v_{k}\right) & \lambda^{\prime}\left(u_{k}\right)=6 p+g+k, & \lambda^{\prime}\left(w_{k}\right)=6 p+g+1-k, \\
\lambda^{\prime}\left(v_{k}^{\prime}\right)=4 p+g+1-k, & \lambda^{\prime}\left(u_{k}^{\prime}\right)=8 p+g+1-k, & & \lambda^{\prime}\left(w_{k}^{\prime}\right)=2 p+g+k, \\
\lambda^{\prime}\left(v_{k}^{\prime \prime}\right)=8 p+g+1-k, & \lambda^{\prime}\left(u_{k}^{\prime \prime}\right)=4 p+g+k, & \lambda^{\prime}\left(w_{k}^{\prime \prime}\right)=2 p+g+1-k, \\
\lambda^{\prime}\left(f_{k}\right)=12 p+g k, & \lambda^{\prime}\left(f_{k}^{\prime}\right)=12 p+g+1-k & \lambda^{\prime}\left(f_{k}^{\prime \prime}\right)=10 p+g+1-k,
\end{array}
$$


$\lambda^{\prime}\left(f_{k}^{\prime \prime \prime}\right)=10 p+g+k$

$\lambda^{\prime}\left(g_{k}\right)=\lambda\left(g_{k}\right), 1 \leq k \leq g$.

The following are the weights for newly added faces:

$$
\begin{gathered}
\mathrm{wt}\left[\lambda^{\prime}\left(f_{k}\right)\right]=\lambda^{\prime}\left(v_{k}\right)+\lambda^{\prime}\left(v_{k}^{\prime}\right)+\lambda^{\prime}\left(v_{k}^{\prime \prime}\right)+\lambda^{\prime}\left(f_{k}\right) \\
=k+4 p+g+1-k+8 p+g+1-k+12 p+g+k=24 p+3 g+2 \\
\mathrm{wt}\left[\lambda^{\prime}\left(f_{k}^{\prime}\right)\right]=\lambda^{\prime}\left(v_{k}\right)+\lambda^{\prime}\left(u_{k}\right)+\lambda^{\prime}\left(w_{k}\right)+\lambda^{\prime}\left(f_{k}^{\prime}\right) \\
=k+6 p+g+k+6 p+g+1-k+12 p+g+1-k=24 p+3 g+2 \\
\mathrm{wt}\left[\lambda^{\prime}\left(f_{k}^{\prime \prime}\right)\right]=\lambda^{\prime}\left(v_{k}^{\prime}\right)+\lambda^{\prime}\left(u_{k}^{\prime}\right)+\lambda^{\prime}\left(w_{k}^{\prime}\right)+\lambda^{\prime}\left(f_{k}^{\prime \prime}\right) \\
=4 p+g+1-k+8 p+g+k+2 p+g+k+10 p+g+1-k=24 p+4 g+2 \\
\mathrm{wt}\left[\lambda^{\prime}\left(f_{k}^{\prime \prime \prime}\right)\right]=\lambda^{\prime}\left(v_{k}^{\prime \prime}\right)+\lambda^{\prime}\left(u_{k}^{\prime \prime}\right)+\lambda^{\prime}\left(w_{k}^{\prime \prime}\right)+\lambda^{\prime}\left(f_{k}^{\prime \prime \prime}\right) \\
=8 p+g+1-k+4 p+g+k+2 p+g+1-k+10 p+g+k=24 p+4 g+2
\end{gathered}
$$

Hence the resultant graph is $(1,0,1)$ face bimagic for all 3 -sided faces with $k_{1}=24 p+3 g+2$ and $k_{2}=24 p+4 g+2$.

\section{Conclusion}

In this paper, the face bimagic labeling of double duplication of all vertices by edges of a ladder graph along with a general result is studied. In future, this labeling technique can be used for real time applications like communication, radar fields etc.

\section{References}

M.A. Ahmed and J. Baskar Babujee, Bimagic labeling for strong face plane graphs, International Conference on Mathematical Computer Engineering, VIT University, Chennai, India, (2015).

J. Baskar Babujee, "Bimagic labeling in path graphs",The Mathematics Education, Volume 38, No. 1, (2004) pp.12-16.

J.A. Gallian, A Dynamic Survey of Graph Labeling, Electronic Journal of Combi- natorics, \#DS6, (2020).

Mohammed Ali Ahmed and J. Baskar Babujee, On Face Bimagic Labeling of Graphs, IOSR Journal of Mathematics, Volume12, Issue 6, (2016) pp. 01-07.

Roopa.B, Shobana.L, On face bimagic labeling for duplication and double dupli- cation graphs, AIP Conference Proceedings 2112, 020155 (2019).

Roopa.B, Shobana.L, On Face Bimagic Labeling for Double Duplication of Tree related Graphs, International Journal of Advanced Science and Technology, 29(04) (2020) pp. 5687-5694.

A. Rosa, On certain valuations of the vertices of a graph, Theory of Graphs, Internat. Symposium, Rome, July 1966, Gordon and Breach, N. Y. and Dunod Paris (1967) pp 349-355. 